Swarthmore College

Works

Physics \& Astronomy Faculty Works

Physics \& Astronomy

$12-15-2000$

\title{
A Two-Chain Path Integral Model Of Positronium
}

L. Larrimore

R. N. McFarland

P. A. Sterne

Amy Lisa Graves

Swarthmore College, abug1@swarthmore.edu

Follow this and additional works at: https://works.swarthmore.edu/fac-physics

Part of the Physics Commons

Let us know how access to these works benefits you

\section{Recommended Citation}

L. Larrimore, R. N. McFarland, P. A. Sterne, and Amy Lisa Graves. (2000). "A Two-Chain Path Integral Model Of Positronium". Journal Of Chemical Physics. Volume 113, Issue 23. 10642-10650. DOI: 10.1063/ 1.1323979

https://works.swarthmore.edu/fac-physics/90

This work is brought to you for free by Swarthmore College Libraries' Works. It has been accepted for inclusion in Physics \& Astronomy Faculty Works by an authorized administrator of Works. For more information, please contact myworks@swarthmore.edu. 


\section{AD| $\begin{aligned} & \text { The Journal of } \\ & \text { Chemical Physics }\end{aligned}$}

\section{A two-chain path integral model of positronium}

L. Larrimore, R. N. McFarland, P. A. Sterne, and Amy L. R. Bug

Citation: The Journal of Chemical Physics 113, 10642 (2000); doi: 10.1063/1.1323979

View online: http://dx.doi.org/10.1063/1.1323979

View Table of Contents: http://scitation.aip.org/content/aip/journal/jcp/113/23?ver=pdfcov

Published by the AIP Publishing

\section{Articles you may be interested in}

Collecting high-order interactions in an effective pairwise intermolecular potential using the hydrated ion concept: The hydration of Cf3+

J. Chem. Phys. 140, 214104 (2014); 10.1063/1.4879549

A refined potential for hydroxylamine clusters and the liquid phase

J. Chem. Phys. 135, 054502 (2011); 10.1063/1.3610344

The solvation of ions in acetonitrile and acetone. II. Monte Carlo simulations using polarizable solvent models J. Chem. Phys. 117, 8467 (2002); 10.1063/1.1512281

Quantum, intramolecular flexibility, and polarizability effects on the reproduction of the density anomaly of liquid water by simple potential functions

J. Chem. Phys. 115, 10758 (2001); 10.1063/1.1418243

Circumventing the pathological behavior of path-integral Monte Carlo for systems with Coulomb potentials J. Chem. Phys. 107, 571 (1997); 10.1063/1.474442

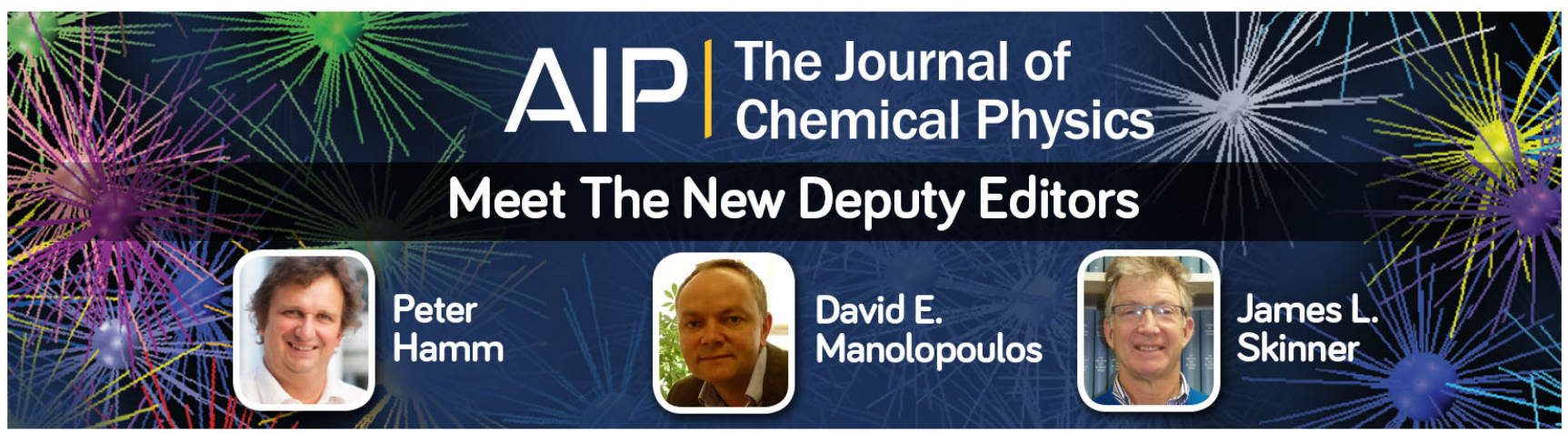




\title{
A two-chain path integral model of positronium
}

\author{
L. Larrimore and R. N. McFarland \\ Department of Physics and Astronomy, Swarthmore College, Swarthmore, Pennsylvania 19081
}

P. A. Sterne

Lawrence Livermore National Laboratory, Livermore, California 94550

Amy L. R. Buga)

Department of Physics and Astronomy, Swarthmore College, Swarthmore, Pennsylvania 19081

(Received 7 August 2000; accepted 19 September 2000)

\begin{abstract}
We have used a path integral Monte Carlo technique to simulate positronium (Ps) in a cavity. The primitive propagator is used, with a pair of interacting chains representing the positron and electron. We calculate the energy and radial distribution function for Ps enclosed in a hard, spherical cavity, and the polarizability of the model Ps in the presence of an electrostatic field. We find that the positron distribution near the hard wall differs significantly from that for a single particle in a hard cavity. This leads to systematic deviations from predictions of free-volume models which treat Ps as an effective, single particle. A virial-type estimator is used to calculate the kinetic energy of the particle in the presence of hard walls. This estimator is found to be superior to a kinetic-type estimator given the interaction potentials, cavity sizes, and chain lengths considered in the current study. (C) 2000 American Institute of Physics. [S0021-9606(00)50447-4]
\end{abstract}

\section{INTRODUCTION}

Positron annihilation spectroscopy is a widely-used experimental probe of solids, liquids, and gases. ${ }^{1}$ Coulomb repulsion with the nuclei makes the positron most sensitive to open-volume regions such as vacancies, voids, and molecular free-volumes. In insulators, positrons often form positronium (Ps), bound hydrogeniclike electron-positron pairs, which diffuse into the open-volume regions. The long intrinsic lifetime of ortho-positronium (o-Ps) is shortened by annihilation with surrounding electrons, resulting in a measurement of the size of the open volume region based on the observed positron lifetime. ${ }^{2}$ Positron annihilation in such a system has been described in terms of free-volume models. ${ }^{3,4}$ A correlation curve relating the positron lifetime to the size of the free volume has been parameterized by Nakanishi et $a .^{5}$ based on measurements of systems with well-defined open volume regions, including molecular crystals and zeolites. This approach is now used extensively in measurements of free volumes for a wide range of insulating systems. $^{2}$

Free-volume models ${ }^{3,4}$ are built on very simple assumptions, including interaction only within some limited distance of the cavity wall, and simple geometric shapes for an "open-volume" region. We can assess the applicability of these simple approximations provided we have a suitable model for the behavior of the quantum mechanical positronium in the system. Path Integral Monte Carlo provides a convenient approach for this. In this paper, we present results of PIMC simulations of positronium treated as a two-particle electron-positron system. We consider the interaction of positronium within a hard-sphere as a simple approximation

a) Author to whom correspondence should be addressed. to a solid environment, and compute the effect of size constraint on the behavior of positronium in an open-volume region in a solid.

In the path integral Monte Carlo (PIMC) technique, ${ }^{6,7}$ a single quantum particle is represented by a polymeric chain of fictitious particles, or "beads." PIMC has lent itself naturally to several studies of Ps trapped within an insulating environment. Reese and Miller ${ }^{8}$ have utilized a single chain of beads, where each bead represents the composite Ps particle in fluid $\mathrm{Xe}$, to determine the annihilation rate and tendency of Ps to localize near the liquid-vapor critical point. Schmitz and Müller-Plathe ${ }^{9}$ have used a similar model to study Ps annihilation within a polystyrene fluid whose monomers are simulated as classical particles interacting with Ps via a semiempirical (Buckingham) potential. While these studies provide much insight, further information may be gained by using a two-particle model of Ps, in which the interaction of the Ps with the host atoms is based on the interactions of its individual constituents, rather than on effective interactions. In addition, it is possible to study the binding energy and ionization of Ps within a two-particle approach.

Recently, ${ }^{10}$ Müser and Berne have proposed an elegant solution to a longstanding problem of simulating Coulombattracting systems with a "primitive" approximation to the propagator used in the path integral. They tested their formalism with a successful simulation of the electron of hydrogen, a single-chain calculation. In the current work, we will use the Müser-Berne scheme to study a stable, twochain path integral representation of Ps. The approach is described in more detail in Sec. II. In Sec. III, we present results using this approach for the positronium distribution, energy, and polarizability in a spherical cavity. We conclude 
that this method provides a simple, flexible approach for simulations of Ps in solids.

\section{COMPUTATIONAL MODEL}

We wish to calculate the thermal averages of quantities related to Ps. For a quantity represented by the operator $\hat{A}$, this amounts to finding

$$
\langle\hat{A}\rangle=\frac{1}{Q} \operatorname{Tr} \hat{\rho} \hat{A} ; Q \equiv \operatorname{Tr} \hat{\rho} .
$$

In the canonical ensemble, the quantum density matrix $\hat{\rho}$ is ${ }^{11}$

$$
\hat{\rho}(\beta)=\exp (-\beta \hat{H}),
$$

with $\beta$ the inverse temperature and $\hat{H}$ the Hamiltonian operator for the entire system held at fixed temperature and volume. In a well-known series of steps ${ }^{12}$ one expresses the trace in Eq. (1) in the position representation, breaks the right-hand side of Eq. (2) into a product of $P \equiv \beta / \epsilon$ terms, and inserts $P-1$ complete sets of position states to obtain

$$
\begin{aligned}
\left\langle\mathbf{R}|\hat{\rho}(\beta)| \mathbf{R}^{\prime}\right\rangle= & \int\left\langle\mathbf{R}|\hat{\rho}(\epsilon)| \mathbf{R}_{1}\right\rangle \\
& \times\left\langle\mathbf{R}_{1}|\hat{\rho}(\epsilon)| \mathbf{R}_{2}\right\rangle \cdots\left\langle\mathbf{R}_{P-1}|\hat{\rho}(\epsilon)| \mathbf{R}^{\prime}\right\rangle \\
& \times d \mathbf{R}_{1} \cdots d \mathbf{R}_{P-1} .
\end{aligned}
$$

While this is exact, the substitution of a suitable hightemperature approximation to $\hat{\rho}(\epsilon)$ in Eq. (3) provides a discretized approximation to $Q$, and thus to

$$
\langle\hat{A}\rangle=\frac{1}{Q} \int d \mathbf{R} d \mathbf{R}^{\prime}\left\langle\mathbf{R}|\hat{\rho}(\beta)| \mathbf{R}^{\prime}\right\rangle\left\langle\mathbf{R}^{\prime}|\hat{A}| \mathbf{R}\right\rangle .
$$

Numerous computational schemes based on Monte Carlo or Molecular Dynamics ${ }^{6,7,13-16}$ have been used to sample the space of paths delineated in Eq. (3). Equation (4) is rarely calculated directly; rather, one constructs an estimator for $\langle\hat{A}\rangle$ which takes advantage of the computational scheme. ${ }^{6,17}$

For our current study of $e^{+}$and $e^{-}$, the position basis is the six-dimensional position space of the two particles, $|\mathbf{R}\rangle$ $\equiv\left|\mathbf{r}^{+} \mathbf{r}^{-}\right\rangle$. Interactions exist both between $e^{+}$and $e^{-}$and with sources imagined to be external to them. Thus, the Hamiltonian may be written as

$$
\hat{H}=\hat{T}_{+}+\hat{T}_{-}+\hat{V}+\hat{U}_{+}+\hat{U}_{-},
$$

with $\hat{T}_{+}, \hat{T}_{-}$the kinetic energies of $e^{+}, e^{-}$. In the position representation, $\hat{V} \equiv V(\mathbf{r})$, where $\mathbf{r}$ is the relative coordinate, and $U_{+}, U_{-}$are functions of $\mathbf{r}^{+}$and $\mathbf{r}^{-}$, respectively. A simple high-temperature approximation puts the density matrix in the pair-product ${ }^{18}$ form

$$
\hat{\rho}(\epsilon) \approx e^{-\epsilon\left(\hat{T}_{+}+\hat{T}_{-}\right)} e^{-\epsilon \hat{V}} \hat{\rho}_{0}(\epsilon) .
$$

It is formally correct to write in this, the primitive approximation, ${ }^{6}$

$$
\hat{\rho}_{0}(\epsilon)=e^{-\epsilon\left(\hat{U}_{+}+\hat{U}_{-}\right)} .
$$

However, the current study will involve an external interaction with hard walls, and will benefit from an alternative treatment of $\hat{\rho}_{0}$. Theoretically, convergence of the partition function has been shown to exist for hard walls in the primitive approximation as $P \rightarrow \infty .{ }^{10}$ But for any practical calculation, the approach of a path integral Monte Carlo result to the correct answer in the presence of hard planar ${ }^{18}$ or curved $^{19}$ walls is slow if one makes the naive substitution, which in our case would be

$$
\left\langle\mathbf{r}^{+} \mathbf{r}^{-}\left|\hat{\rho}_{0}(\epsilon)\right| \mathbf{r}^{\prime+} \mathbf{r}^{\prime-}\right\rangle=\left\{\begin{array}{ll}
1 & r^{+} \text {and } r^{-} \leqslant r_{c} \\
0 & \text { otherwise }
\end{array} .\right.
$$

As an alternative, some studies on systems of hard spheres have used exact numerical values for $\hat{\rho}_{0}$ appropriate for an isolated pair of spheres. ${ }^{20,21}$ Also, various types of image approximation have been used. For example, the exact density matrix for a particle between two hard planar walls was derived by Barker, ${ }^{18}$ and several studies ${ }^{18,19,22,23}$ have used approximations to this form. ${ }^{24}$ An alternative image approximation due to Kalos and Whitlock ${ }^{25}$ is based on the exact density matrix near a single hard wall. This form was used by Reese and Miller, ${ }^{8}$ and we will use this form as well, in order to compare with the best results of Ref. 23, which also treats Ps within a spherical cavity. ${ }^{26}$ Thus, we will define

$$
\left\langle\mathbf{r}^{+} \mathbf{r}^{-}\left|\hat{\rho}_{0}(\boldsymbol{\epsilon})\right| \mathbf{r}^{\prime+} \mathbf{r}^{\prime-}\right\rangle=\left\{\begin{array}{l}
\prod_{*=+,-}\left[1-\exp \left(-\left(r_{c}^{2}-r^{* 2}\right)\left(r_{c}^{2}-r^{\prime * 2}\right) / 2 \epsilon r_{c}^{2}\right)\right] \quad r^{+} \text {and } r^{-} \leqslant r_{c} \\
0 \quad \text { otherwise }
\end{array}\right.
$$

However we should note that an approximation based on a partial wave expansion of the exact wave function has been derived by $\mathrm{Cao}$ and Berne. ${ }^{27}$ It is a theoretically robust form for a system of particles in a hard spherical cavity, and is likely to provide the most rapidly convergent results.

As mentioned in Sec. I, the attractive Coulomb potential,
$V_{c}(\mathbf{r})=-e^{2} / r$, resists a straightforward primitive path integral calculation. The strong singularity at the origin makes it uncertain whether the discretized approximation to $Q$ should converge to the exact $Q$ in the limit $P \rightarrow \infty$. In fact, the approximate $Q$ seems to behave pathologically for any finite $P$, and one sees a collapse of the path integral chain into the 
center of force as computation proceeds. ${ }^{28}$ In the well known path integral study on $e^{-}$in molten $\mathrm{KCl},{ }^{29}$ the authors chose to use a pseudopotential, finite at the origin, to simulate the interaction of $e^{-}$and $K^{+}$. Similarly, studies of Ps in liquids by Miller and co-workers ${ }^{8,30}$ utilized a pseudopotential between Ps and liquid atoms.

In the current work, we have adopted the Müser-Berne scheme, which amounts to applying an effective potential between $e^{+}$and $e^{-}$which converges exponentially fast to the Coulomb potential as a parameter, $a$, is sent to zero. We adopt their specific choice of a Yukawa potential,

$$
V(\mathbf{r})=-\frac{e^{2}}{r}(1-\exp (-r / a)) \text {. }
$$

They have shown ${ }^{10}$ that in the limit $a \rightarrow 0$ and $P \rightarrow \infty$, the effective potential produces the correct Coulomb partition function; and additionally, that the fastest convergence will occur when $a \propto P^{-2 / 3}$. Coulombic results are extrapolated from a series of simulations for different $P$ and $a$.

An alternative strategy would be to employ the exact Coulomb propagator between electron and positron. This is tractable analytically, as Pollock has discussed. ${ }^{31}$ The exact Coulomb propagator has been utilized, for example, to solve problems involving biexcitons in semconductors. ${ }^{32}$ It has also been a powerful technique with which to study pure hydrogen at a wide variety of temperatures and pressures. ${ }^{33}$ The advantage to using a propagator exact for each pair of particles is that the density matrix is very accurate, and one generally needs fewer beads for equivalent accuracy. ${ }^{34} \mathrm{Ar}-$ guments for the primitive approximation are that it is highly tractable and quicker to compute than the analytical form. A primitive approximation may be desirable for including interactions other than electron-positron; the computational advantages to using the exact form for only one part of the problem are not clear.

Equations (3), (6), and (9) imply that we must sample the distribution

$$
\begin{aligned}
\rho\left(\mathbf{R}, \mathbf{R}^{\prime} ; \boldsymbol{\epsilon}\right) \equiv & \left\langle\mathbf{R}|\hat{\rho}(\beta)| \mathbf{R}^{\prime}\right\rangle \\
= & \int d \mathbf{R}_{1} \cdots d \mathbf{R}_{P-1} \prod_{i=1, P} K_{0}\left(\mathbf{R}_{i-1}, \mathbf{R}_{i}, \boldsymbol{\epsilon}\right) \\
& \times \exp \left(-\epsilon V\left(\mathbf{r}_{i}\right)\right) \rho_{0}\left(\mathbf{R}_{i-1}, \mathbf{R}_{i} ; \boldsymbol{\epsilon}\right),
\end{aligned}
$$

where $\mathbf{R}=\mathbf{R}_{0}, \mathbf{R}^{\prime}=\mathbf{R}_{P}$, and $K_{0}$ is the free-particle (kinetic) density matrix,

$$
\begin{aligned}
K_{0}\left(\mathbf{R}, \mathbf{R}^{\prime} ; \boldsymbol{\epsilon}\right)= & \left(\frac{m}{2 \pi \hbar^{2} \boldsymbol{\epsilon}}\right)^{3} \exp \left(-\frac{m}{2 \hbar^{2} \epsilon}\left[\left(\mathbf{r}^{+}-\mathbf{r}^{\prime+}\right)^{2}\right.\right. \\
& \left.\left.+\left(\mathbf{r}^{-}-\mathbf{r}^{\prime-}\right)^{2}\right]\right) .
\end{aligned}
$$

The form of $K_{0}$ is that of two classical polymers with harmonic bonds. The "classical polymer isomorphism" has produced both analytical ${ }^{35}$ and computational progress.

In the notation of Sprik and co-workers, ${ }^{22}$ our method would be considered a "one-stage, image approximation." Sampling was performed on a pair of chains of $P$ beads with $\mathbf{R}_{0} \equiv \mathbf{R}_{P}$. A direct sampling procedure ${ }^{6,19,36}$ was used to gen- erate independent configurations of the chains. This "threading" procedure ${ }^{21}$ relies on an interpolation formula due to Levy, and amounts to removing randomly situated subsections of length $L$ of the $e^{-}$and $e^{+}$chains, $\left\{\mathbf{R}_{j}, \ldots, \mathbf{R}_{j+L}\right\}$, and replacing them with new subsections of length $L$, $\left\{\mathbf{R}_{j}^{\prime}, \ldots, \mathbf{R}_{j+L}^{\prime}\right\}$. The Levy construction ensures that the new configuration is drawn from the distribution $K_{0}\left(\mathbf{R}_{j}^{\prime}, \mathbf{R}_{j+L}^{\prime} ; L \epsilon\right)$. Then, a standard Metropolis Monte Carlo procedure $^{37}$ was used to either accept or reject the new configuration, with an acceptance probability of

$$
\max \left[1, \prod_{i=j, j+L} \frac{\exp \left(-\epsilon V\left(\mathbf{r}_{i}^{\prime}\right) \rho_{0}\left(\mathbf{R}_{i-1}^{\prime}, \mathbf{R}_{i}^{\prime} ; \epsilon\right)\right.}{\exp \left(-\epsilon V\left(\mathbf{r}_{i}\right) \rho_{0}\left(\mathbf{R}_{i-1}, \mathbf{R}_{i} ; \epsilon\right)\right.}\right] .
$$

A standard way to choose $L$ (not the only way; see Sec. III B) is to vary $L$ in order to have a fixed fraction, typically $50 \%$, of accepted moves. This was done every 10 passes. (One pass is an attempted move of both chains.)

\section{RESULTS}

\section{A. Ps in a spherical cavity}

Two issues motivate us to study Ps in a hard, spherical cavity. First, this is a frequently-used free-volume model for the environment of Ps in a porous solid. ${ }^{4,5,38,39}$ That is, Ps lifetime measurements in solid pores are sometimes interpreted by assuming that Ps behaves like a single particle in its ground state in such a cavity; with a uniform annihilation rate with electronic density in a layer at the cavity wall. ${ }^{4}$ Admittedly a great simplification, this provides a useful organizational principle for data from disparate systems. Nevertheless, it neglects the realistic orbital structure of Ps, and the effects that even a hard cavity (much less one with a realistic, molecular structure) would have on it.

Secondly, there is a technical issue: that at any finite temperature there is a finite likelihood of Ps "ionizing" to a continuum state. For the temperatures chosen, we expect its behavior to be ground-state dominated. Nevertheless, one solution to the formal problem is, upon each pass of the simulation, to return the center of mass of the $e^{+}, e^{-}$system to the center of a generously large $\left(r_{c} \gg a_{0}\right)$ cavity.

Figure 1 shows the radial probability density for the relative coordinate,

$$
P(r) \equiv \frac{1}{Q} \int d \mathbf{R}^{\prime} \rho\left(\mathbf{R}^{\prime}, \mathbf{R} ; \beta\right) \delta\left(r^{\prime}-r\right),
$$

which, in simulational terms, is just the likelihood that a bead pair is at a relative distance of $r$. A total of $300 \mathrm{~K}$ passes were used to accumulate statistics for Fig. 1. "Free" Ps is centered in a cavity with $r_{c}=10$ a.u., with $P=600, a$ $=0.132$ a.u., and $\beta=50$ a.u. The solid line is the exact answer for the $1 S$ state of Ps. These data come from a set of simulations with $P$ in the range $P=320-800$ and $a$ $=k P^{-2 / 3}$, where $k=9.36$. (A large number of beads is required in order to keep discretization parameter $\epsilon$ reasonably small for values of $\beta^{-1}$ much less than $3 / 16$, the Ps groundto-first-excited state energy gap.) As suggested by the discussion in Sec. II, increasing $P$ leads to $P(r)$ curves that fall more closely on the $1 S$ curve. The parameters of Fig. 1 do an excellent job of reproducing the ground state wave function. 


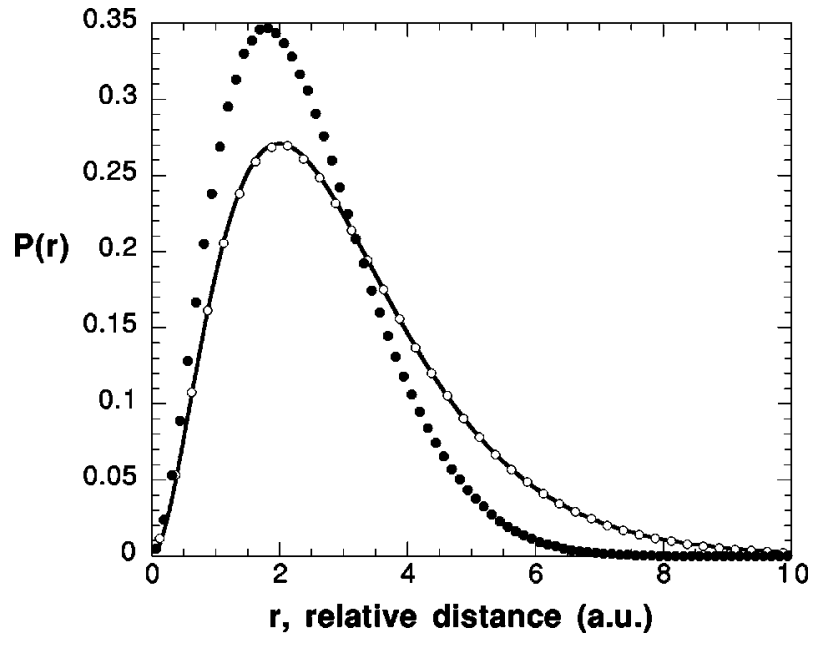

FIG. 1. Radial distribution function, $P(r)$, for Ps in a hard cavity. $r$ is the relative coordinate between electron and positron. Solid line: $1 S$ exact result. Open circles: "Free" Ps, fixed at the center of cavity with radius $r_{c}$ $=10$ a.u. Filled circles: $P$ s in cavity with $r_{c}=5$ a.u. Calculations use Yukawa potential, Eq. (10), with $a=0.132$ a.u., $P=600 ; \beta=50$.

Closed circles in Fig. 1 correspond to Ps which is allowed to equilibrate freely in a spherical cavity with radius $r_{c}=5$ a.u. The effect of the cavity to compress the orbital slightly was noted in Ref. 23. In Table I, we give the location of the maximum of $P(r), r_{\max }$, as a function of cavity radius. The exact, zero-temperature free Ps value is $r_{\max }=2$ a.u., and at $\beta=50, r_{\max }$ will fall between 2.00 and 2.01 a.u. (much closer to the former); 0.01 a.u. is the resolution of our density data.

While, on average, there is no preferred direction for $\mathbf{r}$, there are fluctuations in the shape of the Ps orbital induced by the cavity; since the closest region of hard wall compresses it in a preferred direction. In order to calculate the annihilation rate, one ultimately requires the overlap of the density of $e^{+}$and electrons in the wall. For example, the simple model ${ }^{4}$ presumes a uniform electronic density (more precisely, assumes a uniform annihilation rate when the center of Ps lies) within a layer at the wall. Based on fits to data, the width of the electronic layer can be taken as $\Delta r \approx 3$ a.u. ${ }^{2,5}$ In Table I, we have listed $G(\Delta r)$, the fraction of $e^{+}$ density residing in this layer, for both $\Delta r=3$ and 1 a.u. The $e^{+}$annihilation rate for o-Ps in this simple model would be $\lambda=\lambda_{0} G$, where $\lambda_{0}$ is the annihilation rate in the uniform layer, typically taken as $\lambda_{0}=2 \mathrm{~ns}^{-1}$. 4,40

TABLE I. Calculated values of $r_{\max }$, the location of peak in radial distribution function for Ps in cavity of radius $r_{c}$. Units are a.u. Also listed is calculated likelihood, $G(\Delta r)$, for $e^{+}$to reside within $\Delta r$ of cavity wall. $G$ is calculated from path integral simulation, $G_{\text {th }}$ from simple particle-in-a-box model for $e^{+}$.

\begin{tabular}{cccccc}
\hline \hline$r_{c}$ & $r_{\max }$ & $G(3)$ & $G(1)$ & $G_{\mathrm{th}}(3) / G(3)$ & $G_{\mathrm{th}}(1) / G(1)$ \\
\hline free & 2.01 & $\ldots$ & $\ldots$ & $\ldots$ & $\ldots$ \\
10 & 1.96 & 0.13 & 0.0042 & 1.16 & 1.63 \\
8 & 1.94 & 0.20 & 0.0067 & 1.30 & 1.87 \\
6 & 1.88 & 0.41 & 0.016 & 1.22 & 1.79 \\
5 & 1.79 & 0.62 & 0.028 & 1.11 & 1.75 \\
\hline \hline
\end{tabular}

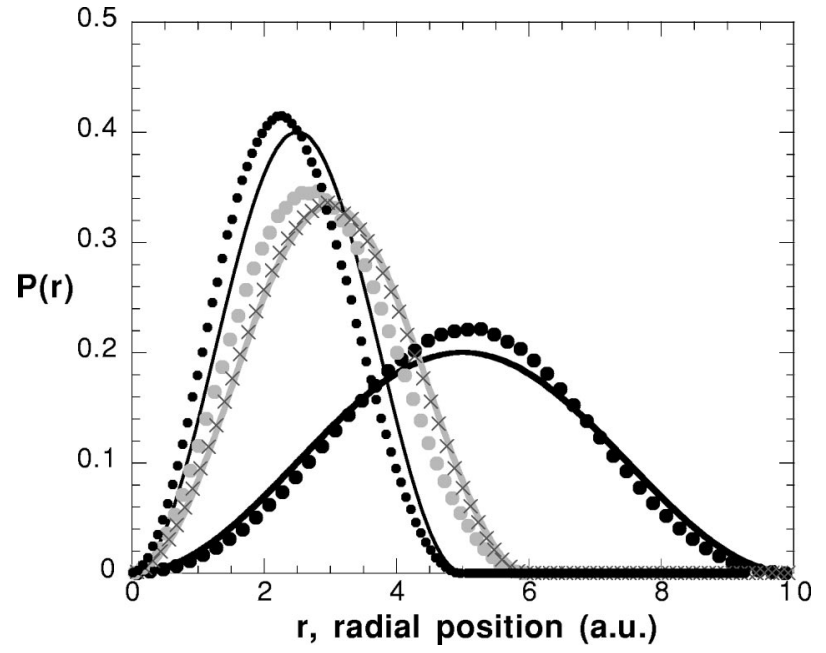

FIG. 2. Distribution function, $P(r)$, of $e^{+}$in Ps, where $r$ is the distance from the center of the cavity. Points are simulated data with parameters as in Fig. 1. Solid lines are particle-in-a-box densities for corresponding values of $r_{c}$. Small black circles: $r_{c}=5$ a.u. Large gray circles: $r_{c}=6$ a.u. Large black circles: $r_{c}=10$ a.u. Crosses: single-particle simulation with $r_{c}=6$ a.u.

Some insight into these annihilation rates comes from viewing the density in the cavity, $P(r)$, as in Fig. 2. The positronic density is close to, but systematically different from, the distribution of a single particle in a hard cavity (a particle-in-a-box). (For reference, a single $e^{+}$particle was simulated with our path integral technique, and as the crosses in Fig. 2 show, these data have the correct distribution.) For the smaller cavities of $r_{c}=5,6$ the positronic position is shifted closer to the cavity center, in agreement with Fig. 3 of Ref. 23. Interestingly, for $r_{c}=10$ a.u., the positronic density is reduced both at the center and at the cavity wall. This leads us to conclude that the common factor driving the shapes of all of these curves is that positronic density is less at the wall than the particle-in-a-box theory predicts. An $e^{-}-e^{+}$bound state orbital must be accommodated next to

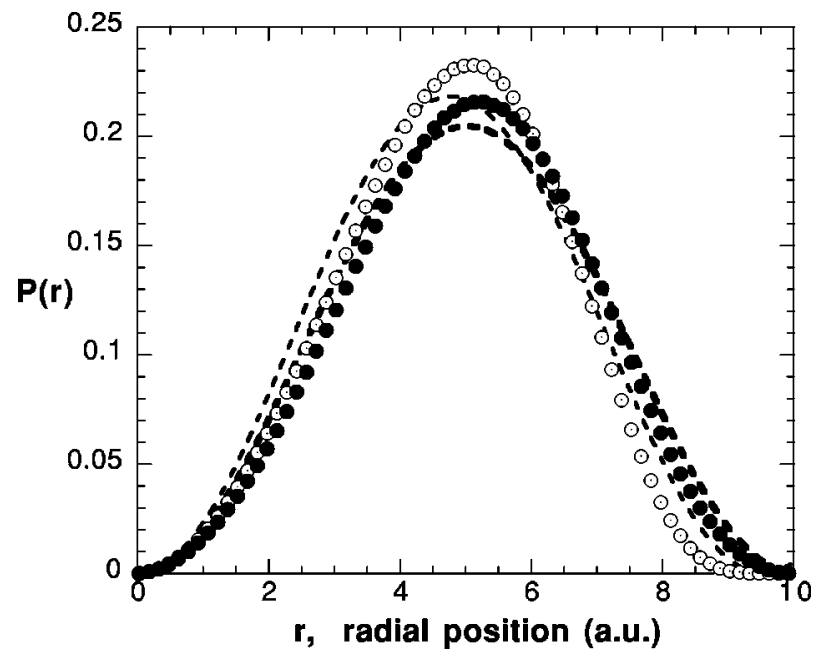

FIG. 3. Distribution function $P(r)$, where $r$ is the distance from the center of the cavity. Runs with $P=600$ averaged over $2 M$ passes, for $\beta=50$ and $r_{c}=10$ a.u. Large black circles: density of $e^{+}$in Ps. Heavy dashed line: fit of these to particle-in-a-box form. Dotted circles: density of the center of Ps. Light dashed line: fit of these to particle-in-a-box form. 
the wall, and this means that the bound positron is more distant from the wall, on average, than an unbound $e^{+}$particle would be. In Table I we list values of $G_{\mathrm{th}} / G$, where $G_{\mathrm{th}}(\Delta r)$ would result if $e^{+}$behaved as a single particle-ina-box. These corrections listed Table I are systematicstemming from the depletion of $e^{+}$density at the wall. They do not vary dramatically with cavity size. We would speculate that they approach a limit as $r_{c}$ increases further, a limit not too different from the values listed in Table I. Nevertheless, a disagreement of as much as thirty percent, as seen in Table I for $\Delta r=3$, would be noticeable on the fits of lifetime vs cavity size in, for example, Fig. 4 of Ref. 2.

Because the $e^{+}$and $e^{-}$are the fundamental moieties in our model of Ps, it is natural to study hard-wall boundary conditions that apply to these bodies. However, as mentioned above, simple pore annihilation models treat the entire Ps atom as the confined particle. Figure 3 shows $P(r)$ for $e^{+}$in Ps, and also for the center of Ps in an $r_{c}=10$ cavity. (This center is not calculated from the pair of centroid positions, but rather, from individual bead pairs.) When the densities in Fig. 3 are fit to the particle-in-a-box form, $P(r)$ $\propto \sin ^{2}\left(\pi r / r_{0}\right)$, a least-squares-fit to the $e^{+}$density yields $r_{0}$ $=10.0$, although the fit is visibly poor. ${ }^{41}$ The fit of the Ps center position is of roughly the same quality; in this case $r_{0}=9.5$. It is reasonable that the effective hard sphere radius for the Ps center-wall interaction would be less than $r_{c}$ by an amount on the order of 1 a.u. Clearly, the effective interaction of the wall with the Ps center would be better fit as a soft, rather than hard, repulsion. Rather than refining this notion further, it is probably more useful to replace the hard cavity with a realistic pore wall geometry, including interactions with the electrons and ions in the solid. This will be the subject of an upcoming study.

\section{B. Energy}

The internal energy $\langle E\rangle$ of a simulated system is frequently of interest (at the very least, as a way to monitor the status of the simulation as it proceeds). The energy of Ps can be estimated in a variety of ways. ${ }^{6}$ We have constructed the "thermodynamic"' estimator, based on

$$
\langle E\rangle \equiv-\frac{1}{Q} \frac{\partial Q}{\partial \beta}=\sum_{i=1}^{P}\left\langle-\frac{\partial}{\partial \beta} \ln \rho\left(\mathbf{R}_{i-1}, \mathbf{R}_{i} ; \epsilon\right)\right\rangle .
$$

For the primitive+image approximation to the Ps density matrix in Eq. (11), this becomes $\langle E\rangle \approx\left\langle T_{\text {kin }}\right\rangle+\left\langle V_{e}\right\rangle$, where the "kinetic" or Barker estimator for the kinetic en$\operatorname{ergy},\langle T\rangle=-(m / \beta Q)(\partial Q / \partial m)$, is ${ }^{18}$

$$
\left\langle T_{\text {kin }}\right\rangle \equiv\left\langle\frac{3 P}{\beta}-\frac{m P}{2} \sum_{*=+,-} \sum_{i=1}^{P}\left(\mathbf{r}_{i-1}^{*}-\mathbf{r}_{i}^{*}\right)^{2} / \hbar^{2} \beta^{2}\right\rangle,
$$

and the potential energy estimator is

$$
\begin{aligned}
\langle V\rangle \approx\left\langle V_{e}\right\rangle \equiv & \frac{1}{P}\left\langle\sum_{i=1}^{P} V\left(r_{i}\right)\right. \\
& \left.-\frac{1}{P} \sum_{i=1}^{P} \frac{\partial \ln \rho_{0}\left(\mathbf{R}_{i-1}, \mathbf{R}_{i} ; \epsilon\right)}{\partial \epsilon}\right\rangle .
\end{aligned}
$$

The second term in Eq. (16) can be thought of as an effective potential energy for interaction with the cavity. It will depend on temperature, growing with increasing $\beta$, but as one would hope, it remains roughly constant for fixed $\epsilon$. For the values of $P$ reported on in this study, it comprises less than $7 \%$ of the energy of a single $e^{+}$particle in an $r_{c}=6$ a.u. cavity at $\beta=50$; and less than $2 \%$ of the energy of Ps under the same conditions.

The error in estimators depends on the method used to estimate and to sample the density matrix, and these dependences have been studied by a number of authors. ${ }^{42-46}$ If the estimated quantity has a measured value of $A(j)$ on the $j$ th measurement pass, then the error in $\langle A\rangle=(1 / N) \sum_{j=1}^{N} A(j)$ after $N$ passes will be

$$
\Delta_{A}=\sqrt{\sigma_{A}^{2} 2 \tau / N} \equiv \delta / \sqrt{N}
$$

with $\sigma_{A}^{2}$ the variance across the entire run,

$$
\sigma_{A}^{2}=\sum_{j=1}^{N}(A(j)-\langle A\rangle)^{2} .
$$

In Eq. (17), $\tau$ is the integrated autocorrelation time, the limit as $n$ becomes large of

$$
\tau(n)=\frac{1}{2}+\sum_{j=1}^{n} C(j)(1-j / N),
$$

where $C(j)$ is the normalized autocorrelation function of the $A(j): \quad C(j)=\left[\langle A(k) A(k+j)\rangle-\langle A\rangle^{2}\right] /\left[\left\langle A^{2}\right\rangle-\langle A\rangle^{2}\right]$. The time $\tau$ is imagined to be a small fraction of the total sampling time, $N$. The kinetic estimator of Eq. (15) has a variance which grows linearly with chain size,

$$
\sigma_{T_{\text {kin }}}^{2} \propto P .
$$

In the face of this drawback, Herman and co-workers ${ }^{43}$ developed a virial estimator for the kinetic energy of a chain moving in a localizing potential $V$, based on this equality of thermodynamic averages,

$$
\left\langle\frac{3 P}{2 \beta}-\frac{P m}{2 \hbar^{2} \beta^{2}} \sum_{i=1}^{P}\left(\mathbf{x}_{i}-\mathbf{x}_{i-1}\right)^{2}\right\rangle=\left\langle\frac{1}{2 P} \sum_{i=1}^{P} \mathbf{x}_{i} \cdot \nabla_{i} V\left(\mathbf{x}_{i}\right)\right\rangle .
$$

Equation (17) suggests that a combination of low variance and quick relaxation will minimize $\delta$, and thus minimize $\Delta_{E}$ for a given number of passes, $N$. Different schemes have been attempted for updating bead positions; ${ }^{44-46}$ and, for example, performance benefitted from updating schemes that reduced serial correlations in $A(j)$ without increasing the variance. Indeed, Janke and $\mathrm{Sauer}^{46}$ have shown how a linear combination of both kinetic and virial estimators will minimize error further; and that staging lengths, hence acceptance rates, might be chosen in order to achieve peak performance. Though quantitative results certainly depend on the potential used, one might summarize the published findings by saying that in the case of a staging algorithm like our own, neither $\tau_{E_{\text {kin }}}, \tau_{E_{\mathrm{vir}}}$ nor $\sigma_{E_{\mathrm{vir}}}^{2}$ were found to vary strongly with $P$.

In the studies mentioned above, single particles moved in potential wells which were analytical functions of posi- 
TABLE II. Calculated energies, in a.u. for Ps in a hard, spherical cavity. Results extrapolated from runs with $P=320,400,510,600$ at $\beta=50,1 \mathrm{M}$ passes per run.

\begin{tabular}{cccc}
\hline \hline$r_{c}$ & $\langle V\rangle$ & $\langle E\rangle$ (Kinetic estimator) & $\langle E\rangle$ (Virial estimator) \\
\hline free & $-0.500(3)$ & $-0.25(1)$ & $-0.252(5)$ \\
10 & $-0.521(3)$ & $-0.24(2)$ & $-0.19(1)$ \\
8 & $-0.528(3)$ & $-0.23(2)$ & $-0.18(1)$ \\
6 & $-0.564(3)$ & $-0.17(1)$ & $-0.132(5)$ \\
5 & $-0.597(2)$ & $-0.11(1)$ & $-0.06(1)$ \\
\hline \hline
\end{tabular}

tion. Many-body path integral calculations have of course also employed virial estimators, but to our knowledge the interaction potentials were again analytical one, two, and higher-body forms. There is no reason that the virial argument cannot be extended to systems with hard walls, to obtain an energy estimator consistent with an effective twobody density matrix as in Eq. (11). A straightforward calculation similar to that of Herman and coworkers leads to this expression for the virial estimate of the kinetic energy of Ps in a cavity,

$$
\begin{aligned}
\left\langle T_{\mathrm{vir}}\right\rangle & =\left\langle\frac{1}{2 P} \sum_{i=1}^{P} r_{i} \frac{\partial V\left(r_{i}\right)}{\partial r_{i}}\right\rangle-F / 2 ; \\
F= & \frac{1}{\beta}\left\langle\sum _ { * = + , - } \sum _ { i = 1 } ^ { P } \mathbf { r } _ { i } ^ { * } \cdot \nabla _ { i } \left(\ln \rho_{0}\left(\mathbf{r}_{i-1}^{*}, \mathbf{r}_{i}^{*} ; \epsilon\right)\right.\right. \\
& \left.\left.+\ln \rho_{0}\left(\mathbf{r}_{i}^{*}, \mathbf{r}_{i+1}^{*} ; \epsilon\right)\right)\right\rangle .
\end{aligned}
$$

The strictest test of the new virial form Eq. (22) is one where there is no interaction potential, but only hard walls which give rise to a kinetic energy. We tested the virial estimator for a single $e^{+}$particle in a cavity. A series of runs with $P=300,450,600,900$ and $P / \beta=6$ yield $\left\langle T_{\text {kin }}\right\rangle$ $=0.124(4),\left\langle T_{\text {vir }}\right\rangle=0.134(3)$, as compared with the exact answer of $\langle T\rangle=0.1371$. The kinetic estimator is systematically low; and it is possible that this is true, but to a much lesser degree, for the virial estimator as well. Equations (15), (16), and (22) were then used to calculate the energy of Ps confined in cavities of various radii. These energies were extrapolated from a set of simulations with increasing $P$ as described in Sec. III A; the kinetic and potential energies were assumed to approach their asymptotic (Coulombic) values as $P^{-4 / 3} \cdot{ }^{10}$ Table II contains these results. For the most part, the potential and kinetic energies are in approximate agreement with earlier results of Liu and Broughton. ${ }^{23}$ Again, there is a trend of lower values from the kinetic estimator, compared with both the virial estimator and data of Ref. 23.

In order to determine the expected error in Monte Carlo energy estimates, it seems appropriate to determine the convergence properties of potential and kinetic energies separately, in case these have different characteristic relaxation behaviors. Consider first the kinetic energy for a single $e^{+}$ particle in a cavity. Figure 4 shows that the variance of the kinetic estimator scales roughly with $P$ as one expects, Eq. (20). The variance of the virial estimator, $\sigma_{T_{\text {vir }}}^{2}$ is not only

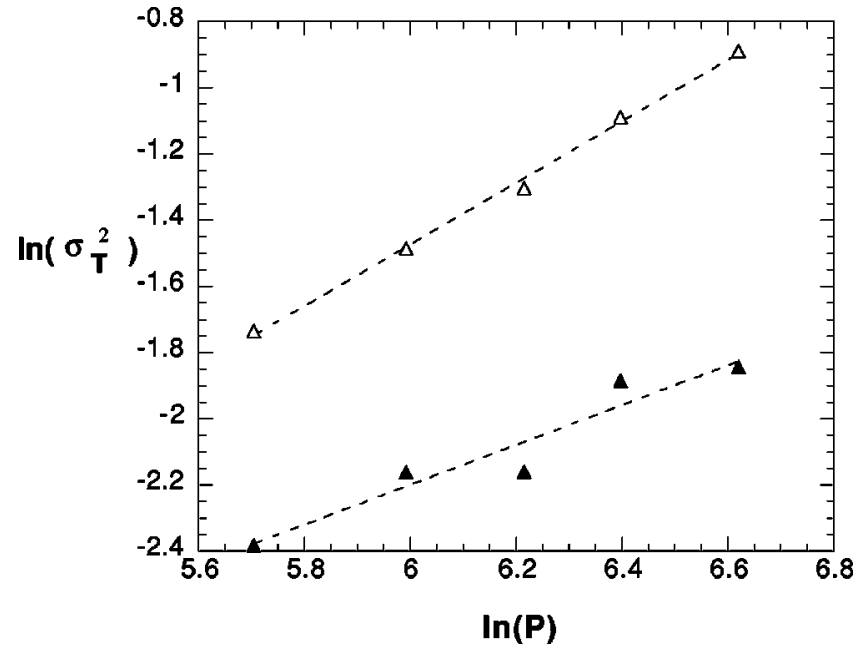

FIG. 4. Natural $\log$ of the variance, $\sigma_{T}^{2}$, of the kinetic energy estimator vs the $\log$ of $P$, for $P=320,400,510,600,750$. Open triangles: $\sigma_{T_{\text {kin }}}^{2}$. Filled triangles: $\sigma_{T_{\text {vir }}}^{2}$. Dashed lines are linear least squares fits to data: slopes are $\alpha=0.93(6), \stackrel{\text { vir }}{0.6(1) .}$

smaller in magnitude for each value of $P$, but also has a weaker $P$ dependence. Equations (9) and (22) suggest that only beads which are in close proximity to the cavity wall will make a large contribution to $\left\langle T_{\text {vir }}\right\rangle$. Table III lists the percentage of beads whose combined energies account for a fixed percentage of the kinetic energy. One can see that while the kinetic estimator "democratically" draws, for example, $99 \%$ of the energy from about $91 \%$ of beads, and that the percentage of beads contributing a fixed percentage does not vary with $P$, the situation is different for the virial estimator. Instead, a small percentage of beads contributes the overwhelming majority of the energy, and this percentage decreases as $P$ increases. In terms of scaling behavior, $\sigma_{T}^{2}$ $\propto P^{\alpha}$, a fit to the data of Fig. 4 yields $\alpha=0.93(6), 0.6(1)$ for the kinetic and virial estimator, respectively. The idea that the virial kinetic energy arises not from the bulk, but from a portion of the surface of the chain makes the exponent value $\alpha \approx 2 / 3$ a reasonable one.

The relaxation behavior of the various estimators is shown in Fig. 5. For the single $e^{+}$, in Fig. 5(a), both the kinetic and virial estimators for $\langle T\rangle$ decorrelate in several time steps, independent of $P$. For the two chains interacting under the Yukawa potential, Fig. 5(b), $\tau$ is longer, but again it is both independent of $P$ and roughly the same for $T_{\text {kin }}(j)$ and $T_{\text {vir }}(j)$. (In order to test the $P$ dependence of $\tau$ in this

TABLE III. Percentage of beads which combine to produce a fixed percentage of the kinetic energy. Single $e^{+}$in a cavity with $r_{c}=6$ a.u.; data averaged over $30 \mathrm{~K}$ passes at $\beta=50$.

\begin{tabular}{ccccccccc}
\hline \hline & \multicolumn{3}{c}{ Kinetic estimator } & & \multicolumn{3}{c}{ Virial estimator } \\
\cline { 2 - 5 } \cline { 6 - 8 } \% of energy & $P=300$ & $P=500$ & $P=700$ & & $P=300$ & $P=500$ & $P=700$ \\
\hline 50 & 22.54 & 22.54 & 22.60 & & 0.085 & 0.035 & 0.029 \\
80 & 50.33 & 50.34 & 50.43 & & 0.330 & 0.148 & 0.100 \\
90 & 65.64 & 65.61 & 65.65 & & 0.557 & 0.252 & 0.167 \\
99 & 90.67 & 90.63 & 90.63 & & 1.553 & 0.653 & 0.436 \\
99.9 & 97.56 & 97.57 & 97.59 & & 2.830 & 1.070 & 0.738 \\
\hline \hline
\end{tabular}




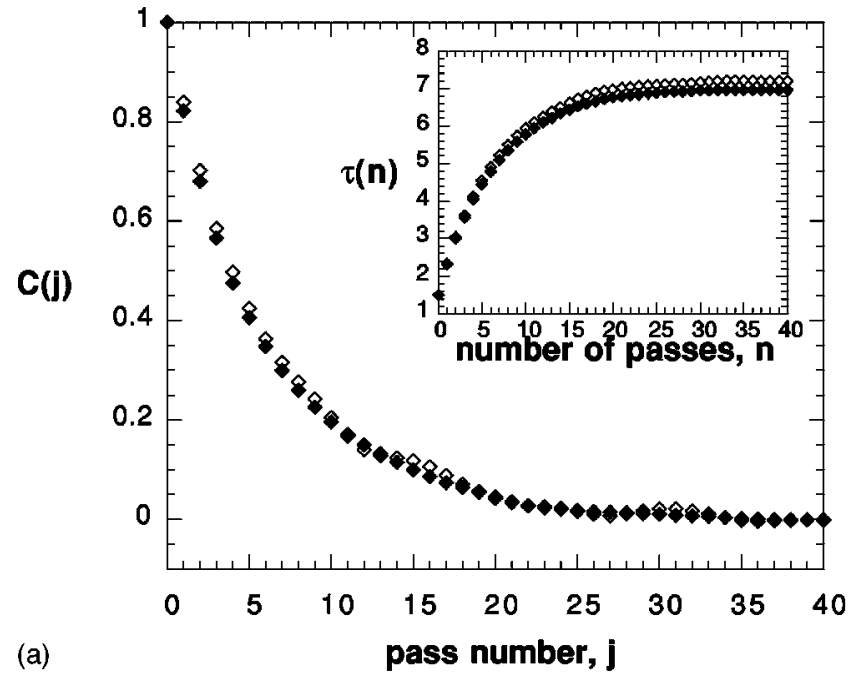

(a)

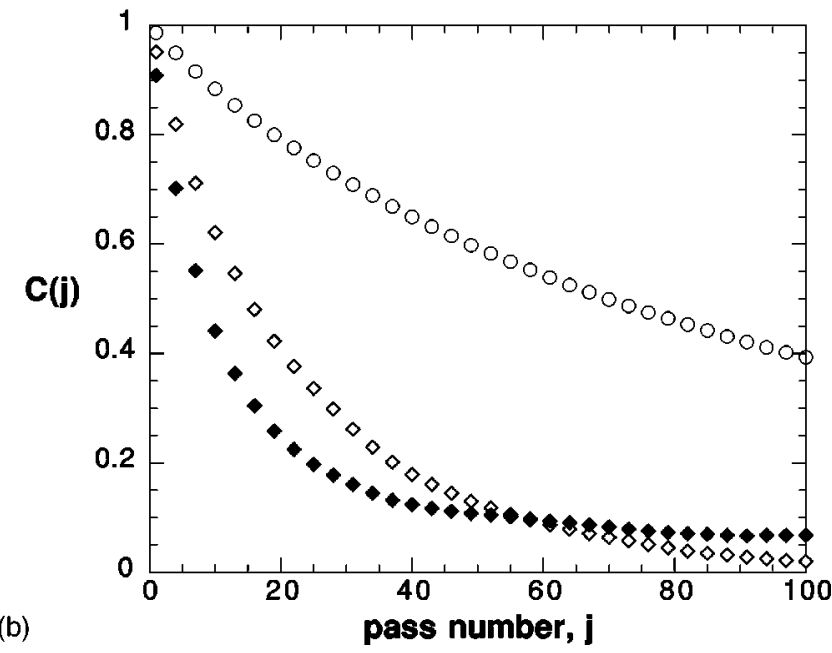

FIG. 5. (a) Autocorrelation function $C(j)$ of energy estimator for $e^{+}$in $r_{c}$ $=6$ cavity. Open diamonds: estimator $T_{\text {kin }}$. Filled diamonds: estimator $T_{\text {vir }}$. Runs with $P=400, \beta=50$. Inset: Integrated relaxation time $\tau$ of Eq. (19). (b) Autocorrelation function $C(j)$ of energy estimator for Ps in $r_{c}=6$ cavity. Open diamonds: estimator $T_{\text {kin }}$. Filled diamonds: estimator $T_{\text {vir }}$. Open circles: estimator $V_{e}(j)$. Runs with $P=400, \beta=50$.

case, the Yukawa radius $a$ was fixed at $a=0.172$.) The potential energy estimator $V_{e}$ relaxes more slowly still. Again, within our ability to detect, this rate does not vary with $P$. In all cases, $\sigma_{T_{\text {vir }}}^{2}<\sigma_{T_{\text {kin }}}^{2}$. Table IV summarizes the integrated autocorrelation times and the error prefactor $\delta$, for kinetic and potential energies of $e^{+}$and of Ps within the cavity. While for Ps the virial estimator is seen to relax more quickly than the kinetic estimator at early times [Fig. 5(b)],

TABLE IV. Relaxation times $\tau$, Eq. (19), and error prefactors $\delta$, Eq. (17), calculated from $350 \mathrm{~K}$ pass runs with $P=400$ and $\beta=50$. Single $e^{+}$and Ps simulated in hard cavity with $r_{c}=6$ a.u.

\begin{tabular}{|c|c|c|c|c|}
\hline \multirow[b]{2}{*}{ Estimator } & \multicolumn{2}{|c|}{$e^{+}$} & \multicolumn{2}{|c|}{ Ps } \\
\hline & $\tau$ & $\delta$ & $\tau$ & $\delta$ \\
\hline$T_{\text {kin }}$ & $7.0(1)$ & $1.75(2)$ & $25(2)$ & $4.9(2)$ \\
\hline$T_{\text {vir }}$ & $7.0(6)$ & $1.20(10)$ & $32(2)$ & $2.6(1)$ \\
\hline$V_{e}$ & $\cdots$ & $\cdots$ & $125(15)$ & $1.3(1)$ \\
\hline
\end{tabular}

its autocorrelation function contains at least one slowlydecaying mode, so that (Table IV) its integrated relaxation time, $\tau$, is larger. In this context, we note that values of $\tau$ in Table IV were derived by using a three-parameter fit suggested by Janke and Sauer, ${ }^{46,47}$ which assumes that at long times the decay is exponential with a time constant $\tau_{\exp }$. We find rough agreement between $\tau$ and $\tau_{\text {exp }}$ for all cases listed in Table IV except for the case of the virial estimator for the Ps kinetic energy. In that case, $\tau_{\exp }$ is quite a bit longer; and it is comparable to $\tau_{\exp }$ for the Ps potential energy.

We conclude that for the systems simulated in our study, use of the virial estimator will result a smaller error, $\Delta_{E}$, for chain lengths greater than $P=300$. Figure 4 can be extrapolated to small $P$, to predict that a crossover to a regime where the kinetic estimator is more efficient occurs at somewhere around $P=30$ for the single particle in the $r_{c}=6$ cavity. This might be compared with a crossover at $P=60$ for the particle in a convex potential using staging in Ref. 46. But there remains the issue of the systematic error present in $\left\langle T_{\text {kin }}\right\rangle$ for a single particle in a cavity. This error may derive from the way chain configurations are sampled-that the image approximation of Eq. (11) serves as an importance function. It is likely that a different importance function (e.g., that of Ref. 27) would produce a differently-biased average. The question is left open for future investigation.

\section{Polarizability}

Zeolites are polar, aluminosilicate solids. Within this type of insulating solid, guest moieties experience strong electrostatic fields. For example, IR spectroscopic experiments and theoretical calculations ${ }^{48}$ have determined that small guest molecules experience field strengths near $1 \mathrm{~V} / \AA$ within zeolite $\mathrm{A}$ (where $1 \mathrm{~V} / \AA \approx 0.02$ a.u.). Zeolite $\mathrm{A}$ has also been the subject of positron annihilation experiments, in which the decay rate of o-Ps (among other signature processes) was determined. ${ }^{38,49}$

To know whether it might be appropriate for such a system, we have tested the two-chain model in a uniform electrostatic field, and measured its linear polarizability, $\alpha$. Of course, if a uniform electrostatic field is applied to Ps, it no longer exists in a true bound state. ${ }^{50}$ If the field, $\mathbf{E}_{0}$, points in the $z$ direction, the Coulomb-Stark potential is $V(\mathbf{r})$ $=-1 / r-E_{0} z$. The potential minimum is approached when $z \rightarrow-\infty$, and there is a saddle point between this global minimum and the local minimum at $r=0$, which occurs along the $z$ axis, at $z=-1 / \sqrt{E_{0}}$ (atomic units). A semiclassical argument for hydrogenic atoms ${ }^{51}$ would say that since the potential at the saddle point is $V_{s}=-2 \sqrt{E_{0}}$, we can expect Ps in its ground state to dissociate at a field strength of roughly $E_{0}=1 / 64 \approx 0.0156$; which is when the unperturbed Coulomb ground state energy exceeds the saddle point energy. Further, one must not neglect the fact that the particles can tunnel through this energy barrier. The rate of dissociation is extremely small for laboratory-strength fields. ${ }^{50,52}$ However, this rate is extremely sensitive to the magnitude of the field, ${ }^{52}$ and the tunneling time may become relevant for fields in zeolitic solids. Our study does not attempt to model the dissociation behavior. We have, as before, "free" Ps 
pinned in the center of an $r_{c}=10$ a.u. cavity, which for the weakest fields we have considered is not even large enough to include the saddle point of the potential surface. Ps particles still remain associated during runs of duration $500 \mathrm{~K}$ steps for electric fields with a strength up to $E_{0}=0.01$. (They were observed to dissociate at significantly higher fields.)

The linear polarizability has theoretical value $\alpha=36$ a.u. Deriving $\alpha$ from the quadratic Stark shift of the energy, $\Delta E \approx \frac{1}{2} \alpha E_{0}^{2}$, is difficult, requiring an extremely large value of $\beta$ in order to resolve this tiny shift. Instead, the polarizablity was inferred from the induced dipole moment, $z_{0}$. For weak fields, $z_{0}=\alpha E_{0}$. When $z_{0}$ is a sizable fraction of the size of the atomic orbital, $a_{0}=2$ a.u., the field is no longer weak enough for the linear theory of polarizability to hold. ${ }^{53}$ (Note that in the linear theory, $z_{0} \approx 0.3 a_{0}$ when $E_{0}=1 / 64$.) Fields in the range $E_{0}=0.002-0.01$ were employed in order to find $\alpha$. The largest dipole moment induced in these calculations was $z_{0}=0.18 a_{0}$. There is yet another length scale which is relevant to the 2-chain model, the Yukawa radius $a$. For fixed $P$ the 2-chain model will polarize/dissociate in an electric field more easily for larger $a$. In runs used to find the polarizability of the two-chain Ps model, it was always the case that $a<0.3 z_{0}$, where $z_{0}$ was determined selfconsistently from the run.

For each value of the electric field, runs were performed for five values, $P=500,600,700,800,900,1000$, with $a$ $=k P^{-2 / 3}$, where $k=7.11$. The polarizability was estimated at each pass by the displacement between corresponding $e^{+}$ and $e^{-}$beads in the direction of the field,

$$
\langle z\rangle \approx \frac{1}{P} \sum_{i=1}^{P} z_{i} .
$$

In order to extrapolate these results to $a \rightarrow 0$, we note that second-order perturbation theory predicts that the induced dipole moment for the Coulomb potential, $\langle z\rangle_{C}$, will differ from $\langle z\rangle$ for the Yukawa potential by a correction term which scales as $a^{2}$. So we extrapolate assuming $\langle z\rangle=\langle z\rangle_{C}$ $+\operatorname{const} P^{-4 / 3}$. This is how the energy is found to scale in Ref. 10, and it is the way the extrapolation was performed in Sec. III B. Typical raw data, for $E_{0}=0.0025$, is shown in Fig. 6. Runs of length $1.5 \mathrm{M}$ passes were divided into blocks of $250 \mathrm{~K}$ passes, so that error bars could be determined. Figure 7 shows the extrapolated values, $\langle z\rangle_{C}$, as a function of $E_{0}$. The slope is found to be $\alpha=33 \pm 3$ which, though not extremely precise, is in good agreement with theory.

Finally, we should note that the polarizability could alternatively have been obtained from an imaginary time correlation function (iTCF) of the beads in the absense of an electric field. That is, if one uses second order perturbation theory to calculate $\Delta E \approx \frac{1}{2} \alpha E_{0}^{2}$, one arrives at

$$
\begin{aligned}
\alpha & \approx 2 e^{2} \sum_{\nu} \frac{\langle 0|z| \nu\rangle\langle\nu|z| 0\rangle}{E_{\nu}-E_{0}} \\
& =2 e^{2} \int_{0}^{\infty}\left\langle 0\left|e^{\tau \hat{H}} \hat{z} e^{-\tau \hat{H}} \hat{z}\right| 0\right\rangle d \tau .
\end{aligned}
$$

Equation (24) can be approximated numerically by autocorrelating the positions of beads separated by an imaginary

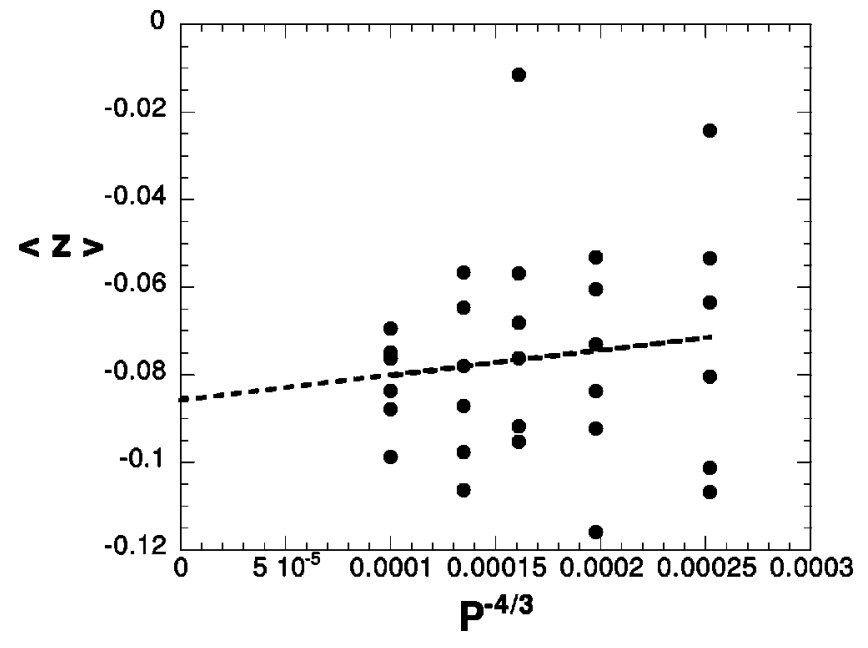

FIG. 6. Raw data of the dipole moment $\langle z\rangle$ as a function of chain length, $P$, for $E_{0}=0.0025$. Each filled circle represents a runs of length $250 \mathrm{~K}$ passes. Result is extrapolated (dashed line) as $\langle z\rangle=\langle z\rangle_{C}+$ const $P^{-4 / 3}$.

time $2 \tau$, and then integrating this iTCF over all such times $\tau$. Such calculations have been performed in the course of quantum Monte Carlo simulations in order to calculate both static and dynamic polarizabilities. ${ }^{54}$

\section{CONCLUSIONS}

We have shown that a path integral Monte Carlo simulation involving a Yukawa approximation to the Coulomb primitive propagator is a simple, effective way to simulate Ps entrapped within a spherical pore. The positions of the beads allow one to visualize the spatial orbital of the Ps atom. There are systematic differences in the distribution of positronic density as compared with the corresponding free volume model. Assuming a uniform electronic density at the pore walls, these result in systematically smaller annihilation rates. The calculated polarizability of Ps is in good agreement with theoretical expectations. Binding energies, calculated with both kinetic and virial estimators, diminish as the radius of the cavity decreases. For large numbers of beads, the greater computational efficiency of the virial estimator is

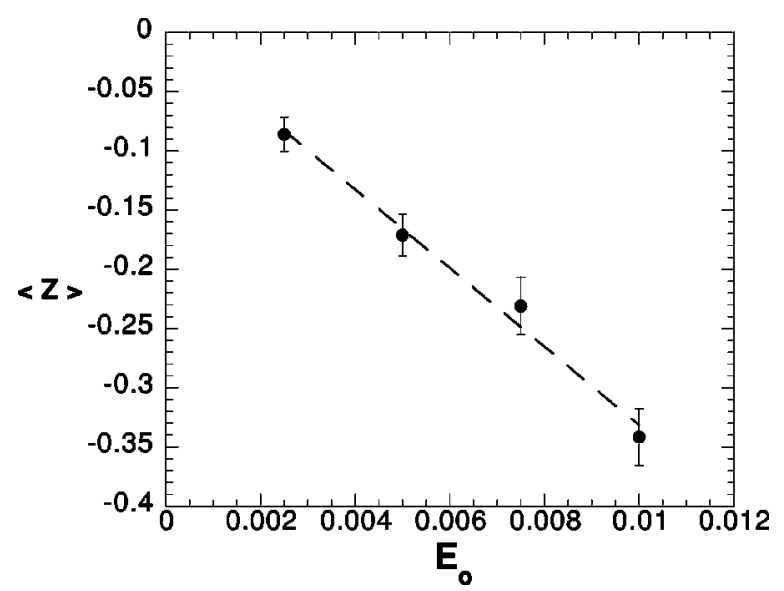

FIG. 7. Extrapolated data $\langle z\rangle_{C}$, as a function of $E_{0}$ fit (dashed line) to the form $\langle z\rangle_{C}=\alpha E_{0}$, yielding $\alpha=33 \pm 3$. 
confirmed. In summary, this approach offers a simple, versatile model on which to base simulations of Ps within insulating solids.

\section{ACKNOWLEDGMENTS}

We are grateful for conversations with G. Martyna, B. Miller, R. Pollock, and T. Reese. We also thank students Gabriel Benjamin-Fernandez of Swarthmore College and Amanda Bonfitto of Earlham College for their collaboration. Acknowledgment is made to the Donors of The Petroleum Research Fund, administered by the American Chemical Society, for support of this research. Additional support was provided by the Faculty Research Support Fund and the Division of Natural Sciences of Swarthmore College. This work was performed under the auspices of the U.S. Department of Energy by University of California Lawrence Livermore National Laboratory under Contract No. W-7405Eng-48.

${ }^{1}$ See, for example, Positron Spectroscopy of Solids, edited by A. Dupasquier and A. P. Mills, Jr. (IOS, Amsterdam, 1995); Proceedings of the 11th National Conference on Positron Annihilation, edited by Y. C. Jean, M. Eldrup, D. M. Schrader, and R. N. West (Trans Tech Publications, Zurich, 1997).

${ }^{2}$ Y. C. Jean, in Positron Spectroscopy of Solids, edited by A. Dupasquier and A. P. Mills, Jr. (IOS, Washington, D.C., 1995).

${ }^{3}$ W. Brandt, S. Berko, and W. W. Walker, Phys. Rev. 12, 1289 (1960).

${ }^{4}$ S. J. Tao, J. Chem. Phys. 56, 5499 (1972); M. Eldrup, D. Lightbody, and J. N. Sherwood, Chem. Phys. 63, 51 (1981); For larger pores, a classical expression for Ps density has also been employed, as in K. Ito, H. Nakanishi, and Y. Ujihira, J. Phys. Chem. B 103, 4555 (1999).

${ }^{5}$ H. Nakanishi, S. J. Wang, and Y. C. Jean, in Positron Annihilation Studies of Fluids, edited by S. C. Sharma (World Scientific, Singapore, 1988).

${ }^{6}$ D. M. Ceperley, Rev. Mod. Phys. 67, 279 (1995).

${ }^{7}$ K. E. Schmidt and D. M. Ceperley, in Monte Carlo Methods in Condensed Matter Physics, edited by K. Binder (Springer, Heidelberg, 1992), p. 205.

${ }^{8}$ T. Reese and B. N. Miller, Phys. Rev. E 47, 2581 (1993).

${ }^{9}$ H. Schmitz and F. Müller-Plathe, J. Chem. Phys. 112, 1040 (2000).

${ }^{10}$ M. H. Müser and B. J. Berne, J. Chem. Phys. 107, 571 (1997).

${ }^{11}$ D. A. McQuarrie, Statistical Mechanics (Harper and Row, New York, 1973), Chap. 10.

${ }^{12}$ T. Pang, An Introduction to Computational Physics (Cambridge University Press, Cambridge, 1997), p. 276; K. Binder, in Encyclopedia of Applied Physics (VCH, New York, 1994), Vol. 10, p. 567; R. P. Feynman, Statistical Mechanics (Benjamin Cummings, Reading, 1972), Chap. 3.

${ }^{13}$ M. P. Allen and D. J. Tildesley, Computer Simulation of Liquids (Clarendon, Oxford, 1987), Chap. 10.

${ }^{14}$ D. L. Freeman and J. D. Doll, Adv. Chem. Phys. 70, 139 (1988).

${ }^{15}$ B. J. Berne and D. Thirumalai, Annu. Rev. Phys. Chem. 37, 401 (1986).

${ }^{16}$ G. J. Martyna, A. Hughes, and M. E. Tuckerman, J. Chem. Phys. 110, 3275 (1999); M. E. Tuckerman, B. J. Berne, G. J. Martyna, and M. L. Klein, ibid. 99, 2796 (1993); R. W. Hall and B. J. Berne, ibid. 81, 3641 (1984).

${ }^{17}$ K. Binder, in Monte Carlo Methods in Statistical Physics, edited by K. Binder (Springer-Verlag, New York, 1979), Chap. 1.

${ }^{18}$ J. A. Barker, J. Chem. Phys. 70, 2914 (1979).

${ }^{19}$ G. Jacucci and E. Omerti, J. Chem. Phys. 79, 3051 (1983).

${ }^{20}$ S. Y. Larsen, J. Chem. Phys. 48, 1701 (1968).
${ }^{21}$ E. L. Pollock and D. M. Ceperley, Phys. Rev. B 30, 2555 (1984).

${ }^{22}$ M. Sprik, M. L. Klein, and D. Chandler, J. Chem. Phys. 83, 3042 (1985).

${ }^{23}$ Z-H. Liu and J. Broughton, Phys. Rev. B 40, 571 (1989).

${ }^{24}$ In Ref. 10, a systematic study of the convergence of the energy with $P$ is performed for this approximation.

${ }^{25}$ P. A. Whitlock and M. H. Kalos, J. Comput. Phys. 30, 361 (1979).

${ }^{26}$ This reference describes a study of Ps in a hard cavity via the method of matrix-squaring, or numerical matrix multiplication. [See A. D. Klemm and R. G. Storer, Aust. J. Phys. 26, 43 (1973); D. Thirumalai, E. J. Bruskin, and B. J. Berne, J. Chem. Phys. 76, 5150 (1982).] A partial-wave expansion is used in this reference to lower the effective dimensionality of the problem.

${ }^{27}$ J. Cao and B. J. Berne, J. Chem. Phys. 97, 2382 (1992).

${ }^{28}$ S. V. Lawande, C. A. Jensen, and H. L. Sahlin, J. Comput. Phys. 3, 416 (1969).

${ }^{29}$ M. Parrinello and A. Rahman, J. Chem. Phys. 80, 860 (1984).

${ }^{30}$ J. Chen and B. N. Miller, Phys. Rev. B 49, 15615 (1994); B. N. Miller, J. Chen, T. L. Reese, and G. Worrell, J. Phys. IV 3, 31 (1993).

${ }^{31}$ E. L. Pollock, Comput. Phys. Commun. 52, 49 (1988).

${ }^{32}$ E. L. Pollock and S. W. Koch, J. Chem. Phys. 94, 6776 (1991); E. L. Pollock and K. J. Runge, ibid. 96, 674 (1992).

${ }^{33}$ C. Pierleoni, D. M. Ceperley, B. Bernu, and W. R. Magro, Phys. Rev. Lett. 73, 2145 (1994); B. Militzer, W. Magro, and D. Ceperley, Contrib. Plasma Phys. 39, 151 (1999).

${ }^{34}$ M. Boninsegni, C. Pierleoni, and D. M. Ceperley, Phys. Rev. Lett. 72, 1854 (1994).

${ }^{35}$ D. Chandler and P. G. Wolynes, J. Chem. Phys. 74, 4078 (1981).

${ }^{36}$ L. D. Fosdick and H. F. Jordan, Phys. Rev. 143, 58 (1966).

${ }^{37} \mathrm{H}$. Gould and J. Tobocknik, An Introduction to Computer Simulation Methods: Applications to Physical Systems (Addison-Wesley, New York, 1996), Chap. 11; J. P. Valleau and S. G. Whittington, in Statistical Mechanics: Part A, edited by B. J. Berne (Plenum, New York, 1977), Chap. 4.

${ }^{38}$ S. Surapanani, M. E. Mullins, and J. T. Waber, J. Am. Ceram. Soc. 76, 292 (1993).

${ }^{39}$ H. Nakanishi and Y. Ujihira, J. Phys. Chem. 86, 4446 (1982); A. Baranowski, M. Debowska, K. Jerie, A. Jezierski, and M. Sachanbinski, Acta Phys. Pol. A 88, 29 (1995).

${ }^{40}$ A. P. Mills, Jr., Phys. Rev. Lett. 46, 717 (1981); G. Ferrante, Phys. Rev. 170, 76 (1968).

${ }^{41}$ Fit fails to pass the $\chi^{2}$ test at the level of $P=0.005$.

${ }^{42}$ A. Giansanti and G. Jacucci, J. Chem. Phys. 89, 7454 (1988).

${ }^{43}$ M. F. Herman, E. J. Bruskin, and B. J. Berne, J. Chem. Phys. 76, 5150 (1982).

${ }^{44}$ J. Cao and B. J. Berne, J. Chem. Phys. 91, 6359 (1989).

${ }^{45}$ W. Janke and T. Sauer, Chem. Phys. Lett. 263, 488 (1996).

${ }^{46}$ W. Janke and T. Sauer, J. Chem. Phys. 107, 5821 (1997).

${ }^{47}$ W. Janke and T. Sauer, J. Stat. Phys. 78, 759 (1995).

${ }^{48}$ A. Zechhina and C. Otero Arean, Chem. Soc. Rev. 25, 187 (1996); H. Bose and H. Forster, J. Mol. Struct. 218, 393 (1990); E. Cohen de Lara and T. Nguyen Tan, J. Phys. Chem. 80, 1917 (1976).

${ }^{49}$ M. B. Perkal and W. B. Walters, J. Chem. Phys. 53, 190 (1970).

${ }^{50}$ L. I. Schiff, Quantum Mechanics (McGraw-Hill, New York, 1968), Chap. 8.

${ }^{51}$ T. F. Gallagher, Rydberg Atoms (Cambridge University Press, Cambridge, 1994), Chap. 1.

${ }^{52}$ J. R. Oppenheimer, Phys. Rev. 13, 66 (1928).

${ }^{53}$ N. Bloembergen, Nonlinear Optics (Addison-Wesley, Menlo Park, 1991), Chap. 1.

${ }^{54}$ B. L. Hammond, W. A. Lester, Jr., and P. J. Reynolds, Monte Carlo Methods in Ab Initio Quantum Chemistry (World Scientific, New Jersey, 1994). 